\title{
Identification of novel mutations of ovarian cancer- related genes from RNA-sequencing data for Japanese epithelial ovarian cancer patients
}

\author{
Saya Nagasawa ${ }^{1), 2)}$, Kazuhiro Ikeda ${ }^{1)}$, Kuniko Horie-Inoue ${ }^{1)}$, Sho Sato ${ }^{3)}$, Satoru Takeda ${ }^{2)}$, Kosei Hasegawa $^{3)}$ \\ and Satoshi Inoue ${ }^{1), 4)}$ \\ 1) Division of Gene Regulation and Signal Transduction, Research Center for Genomic Medicine, Saitama Medical University, \\ Hidaka, 350-1241 Saitama, Japan \\ 2) Department of Obstetrics and Gynecology, Juntendo University, Bunkyo-ku, 113-8431 Tokyo, Japan \\ 3) Department of Gynecologic Oncology, Saitama Medical University International Medical Center, Hidaka 350-1298 Saitama, Japan \\ 4) Department of Systems Aging Science and Medicine, Tokyo Metropolitan Institute of Gerontology, Itabashi-ku 173-0015 Tokyo, \\ Japan
}

\begin{abstract}
Ovarian cancer has the highest mortality rate among gynecological cancers. Gene mutations are involved in the carcinogenesis, metastasis, and therapeutic response in ovarian cancer. However, the variety and proportion of gene mutation is not fully analyzed in Japanese ovarian cancer patients, especially, in those with recurrent tumors. In the present study, RNAsequencing was performed for 32 clinical ovarian specimens obtained from 24 Japanese patients $(24$ primary cancer specimens and 8 recurrent specimens paired with corresponding primary cancer specimens). Mutations in 24 primary specimens were analyzed by comparing the sequence data mapped on RefSeq genes with those in the public online databases BRCA Exchange, COSMIC, ClinVar, and cBioportal. Mutations were observed in TP53 in 16 specimens (67\%), BRCA1 in 9 (38\%), BRCA2 in 13 (54\%), ARIDIA in $3(13 \%)$, PIK3CA in $2(8 \%)$, KRAS in $1(4 \%)$, PTEN in $1(4 \%)$, and CTNNBI in 1 (4\%), excluding synonymous mutations. Among those identified muations, 13 of 14 mutations in TP53, 10 of 11 mutations of $B R C A 1,10$ of 23 mutation positions of BRCA2, none of 7 mutations of ARIDIA, 1 mutation of PIK3CA, and 1 mutation of $C T N N B 1$ were consistent with those reported in the public online databases; however, the other mutations identified were novel. Comparison between matched-paired specimens of primary and recurrent tumors revealed the changes of mutational status in expressed RNAs. RNA-sequencing-based mutation analysis will be useful to reveal ethnic differences of gene mutations in ovarian cancer and to understand the contribution of gene mutations to recurrence.
\end{abstract}

Key words: Ovarian cancer, Mutation, RNA-sequencing, Clear cell carcinoma, High-grade serous carcinoma

OVARIAN CANCER has the highest mortality rate in gynecological cancers and is responsible for the death of approximately 4,500 women per year in Japan [1]. Epithelial ovarian cancer (EOC) is pathologically classified according to the histology as high-grade and low-grade serous carcinoma, clear cell carcinoma (CCC), endometrioid carcinoma (EC), and mucinous carcinoma (MC). High-grade serous carcinoma (HGSC) is the most common histotype of ovarian cancer and is usually diagnosed at an advanced stage, exhibiting aggressive behavior [2]. HGSC occurs in $70 \%$ of malignant ovarian cancer in

Submitted Jul. 4, 2019; Accepted Oct. 21, 2019 as EJ19-0283 Released online in J-STAGE as advance publication Nov. 19, 2019 Correspondence to: Satoshi Inoue, Department of Systems Aging Science and Medicine, Tokyo Metropolitan Institute of Gerontology, 35-2 Sakae-cho, Itabashi-ku, 173-0015, Tokyo, Japan.

E-mail: sinoue07@gmail.com
Caucasian [3]. The characteristics of ovarian cancer among Japanese is rather unique compared with those among Caucasian. Although the proportion of HGSC in Japanese (35\%) is lower than that in Caucasian, the proportion of CCC is as high as $24 \%$ in Japanese (the second highest proportion of the disease) and only $10 \%$ in Caucasian [4]. The proportions of EC (17\%) and MC $(11 \%)$ are also higher in Japanese than those in Caucasian. The difference between histotype proportions of Caucasian and Japanese is possibly caused by the difference of genetic background. The precise mechanism, however, still remains to be clarified.

Somatic mutations in oncogenes have been observed in many human cancers [5]. The Cancer Genome Atlas (TCGA) reported mutations in TP53, which are predominant among ovarian cancer, occurring in at least $96 \%$ of HGSC specimens; approximately $22 \%$ of tumors exhibit- 
ed mutations in BRCA1 and BRCA2 owing to a combination of germline and somatic mutations [6]. Interestingly, exome analysis for Japanese HGSC patients presented TP53 mutation ratio as only $76 \%$ [7]. On the other hand, the prevalence of pathogenic germline $B R C A 1 / 2$ variants in Japanese ovarian cancer patients was not reported to show a large difference compared with that in other ethnicities [8,9]. BRCA1 and BRCA2 play key roles in homologous recombination (HR), which is the primary mechanism for repairing double-stranded DNA breaks. Presumably, HGSC originates from the secretory epithelial cells that have lost their DNA repair systems in the fallopian tubes, whereas CCC and EC can originate from endometriosis. The pathogenesis of endometriosis is multifactorial; however, it may be caused by transtubal dissemination into the peritoneal cavity, leading to endometrial tissue implantation and sustained growth. Pearce et al. reported that endometriosis was associated with a significant risk of CCC; $20.2 \%$ of patients with CCC reported a history of endometriosis [10]. CCC and EC tend to have wild-type TP53 and rarely harbor BRCA1/BRCA2 mutations. In CCC, mutations of the AT-rich interactive domain A (ARID1A) and/or phosphatidylinositol-4,5bisphosphate 3-kinase catalytic subunit $\alpha(P I K 3 C A)$ are commonly observed in Caucasian [11]. Japanese ovarian CCC patients were reported to have higher mutation ratio of ARIDIA (67-78\%) compared to Caucasian, while similar or lower mutation ratio of PIK3CA (11-50\%) compared to Caucasian $[12,13]$. In EC, aberrations have been reported in the Wnt signaling pathway involving the somatic mutations of catenin $\beta 1$ (CTNNB1) and phosphatase and tensin homologue (PTEN) [14]. Ovarian endometrioid carcinoma was reported to harbor mutations of CTNNB1 in $16-53 \%, P T E N$ in $14-20 \%$, and $A R I D 1 A$ in $30-55 \%$ of the cases in Europe [15]. In contrast, mutation ratios of PTEN and ARID1A among Japanese ovarian EC patients are high as $54 \%$ and $92 \%$, respectively [13]. Mutation ratio of CTNNB1 among Japanese EC patients remains to be examined. MC is thought to be malignantly transformed from borderline mucinous tumor, and the most common molecular lesions are observed in the proto-oncogene KRAS (a GTPase [KRAS] mutation); this has been consistently identified in $50-65 \%$ of patients with $\mathrm{MC}[16,17]$. Among Japanse patients, KRAS mutation ratio in MC cases was similar to that among Caucasian whereas that in CCC cases $(25.9 \%)$ was much higher than Caucasian $(7 \%)[12,18]$.

Only a few number of reports is found concerning the genetic status of recurrent ovarian cancer tumors. In a whole exome-sequencing study in paired primary and recurrent samples from 50 HGSC patients with adjuvant platinum-based chemotherapy, BRCA1/2 mutations were maintained between primary and recurrent tumors, except for one pair in which a $B R C A 1$ reversion mutation was identified in the recurrent sample [19]. In a recent whole exome-sequencing study in paired primary and metastatic/recurrent tumors from 57 ovarian cancer patients including mainly HGSC, known cancer driver mutations such as BRCA1/2, TP53, and PIK3CA were also commonly retained during ovarian cancer evolution and/or chemotherapy exposure [20]. They found that primary tumors transmit $54 \%$ of mutations to their corresponding recurrent tumors. Their $\mathrm{CNV}$ analysis also revealed that $3 q 26,8 q 23-24$, and $14 q 11$ were the most significantly amplified regions across all ovarian cancer groups, and PIK3CA amplifications from $3 \mathrm{q} 26$ and $c$ MYC amplifications from 8q23-24 were found in some recurrent patients. In a mutation study for BRCA2mutated ovarian cancers, recurrent ovarian cancer that develops cisplatin resistance would harbor secondary $B R C A 2$ mutations that correct the frameshift caused by mutated $B R C A 2$ alleles, leading to the acquisition of cisplatin resistance [21].

Compared to the DNA-based exome sequencing, RNA-sequencing analysis is capable of providing information regarding the genes that are currently expressed. However, as mentioned above, there are few reports of RNA-sequencing-based mutation analysis. It is also scarcely investigated whether gene mutation rates or mutations positions of Japanese patients are consistent with online databses based on Caucasian. In the present study, we employed high-throughput RNA-sequencing to identify gene mutations in Japanese ovarian cancer patients. RNA-sequencing followed by the mutation analysis of 32 clinical ovarian specimens obtained from 24 patients (24 primary cancer specimens and 8 recurrent specimens paired with corresponding primary cancer specimens) identified novel mutations in TP53, BRCA1, BRCA2, ARID1A, PIK3CA, KRAS, PTEN, CTNNB1, and other HR-related genes such as $A T M$. RNA-sequencingbased mutation analysis will be useful to determine the contribution of gene alteration in ovarian cancer and to understand ethnic differences in mutations.

\section{Materials and Methods}

\section{Clinical specimens}

Experiments using patient data and tissue specimens were approved by the Institutional Review Board of Saitama Medical University International Medical Center (\#13-165). Tumor specimens were available from 24 patients who underwent surgery for the following primary ovarian cancers: primary HGSC (15 specimens, S1-S15), primary CCC (6 specimens, C1-C6), primary unclassifted carcinomas (UCs; 2 specimens, U1 and U2), 
primary EC (1 specimen, E1). Recurrent tumors ( 8 specimens: S11R-S15R, U1R, U2R and E1R) paired with the corresponding primary tumors (S11-S15, U1, U2, and E1) were obtained from surgery or ascites.

\section{RNA-sequencing}

RNA was extracted from freshly frozen tissues using NucleoSpin RNA (Takara, Japan). Quality control was performed using a Bioanalyzer (Agilent, USA), and all RIN (RNA Integrity Number) values were $>8$. RNA library was prepared using the SureSelect Strand Specific RNA Library Prep Kit (Agilent), and 100-bp paired-end RNA-sequencing was performed using the HiSeq $2500^{\circledR}$ (Illumina, USA). rRNA and adapter sequence were deleted from the FASTQ data. RNAsequencing reads were aligned to the human genome assembly hg19 using TopHat based on the Bowtie algorithm, and the mutation positions were identified based on the RefSeq gene model. Gene duplication and raw variants were filtered using the GATK Best Practice and SNPiR systems (available at https://www.broadinstitute. org/gatk/img/BP_workflow_RNA_3.6.png). SNVs specific to the Japanese population were removed according to the Tohoku University database Integrative Japanese Genome Variation.

\section{Mutation analysis}

Mutation data based on RNA-sequencing were compared with those in the online somatic mutation databases BRCA Exchange (available at https://brcaexchange.org/), COSMIC (available at https://cancer.sanger.ac.uk/cosmic) and cBioportal (available at https://www.cbioportal.org/). Mutations were also compared between 8 matchedpaired primary and recurrent tumor samples. Synonymous mutations were excluded from these analysis. We preformed Combined Annotation-Dependent Depletion (CADD) analysis, which is a prediction tool integrating the information from many various functional gene annotations and condensing this information into a single score [22]. High CADD score indicates a potentially deleterious role of the variant. We did not exclude benign variants and variants of unknown significance.

\section{Results}

\section{Mutation analysis for primary ovarian cancer specimens from $R N A$-sequencing data reveals novel mutations}

First, RNA-sequencing was performed for 24 primary ovarian cancer specimens: $15 \mathrm{HGSCs}, 6 \mathrm{CCCs}, 1 \mathrm{EC}$, and 2 UCs (Supplementary Table 1). Then, gene mutations observed in TP53, BRCA1, BRCA2, ARID1A, PIK3CA, KRAS, PTEN, and CTNNB1 were compared with the mutations in these genes reported in the online databases (Fig. 1a) as they were the common mutated genes in ovarian cancer. Mutations were observed in TP53 in 16 specimens (67\%), BRCA1 in 9 (38\%), $B R C A 2$ in $13(54 \%), A R I D 1 A$ in $3(13 \%), P I K 3 C A$ in 2 $(8 \%), K R A S$ in $1(4 \%), P T E N$ in $1(4 \%)$, and CTNNB1 in $1(4 \%)$. The mutation rates in each histotype are shown in Fig. 1b. All data shown in the present study are excluding synonymous mutations.

TP53 mutations were found in 12 of 15 primary HGSCs $(80 \%)$, 1 of 6 primary CCCs $(17 \%), 1$ primary EC (100\%), and 2 primary UCs (100\%) (Table 1). The mutation positions in TP53 identified in primary tumors are shown in Fig. 2a and Table 2. Eleven mutation positions were detected in primary tumors, and ten of them were consistent with those reported in the public online database COSMIC or ClinVar: codons 11, 72, 141, 175, 193, 220, 245, 273, and 378 (underlined in Table 2). Notably, R273H which has been known as a hotspot of mutated TP53 was also detected in the present study. We found a new mutation $\mathrm{R} 267 \mathrm{G}$ with a high Combined Annotation Dependent Depletion (CADD) score similar to the level of known mutations such as G245S, R175H, and $\mathrm{Y} 220 \mathrm{C}$, indicating that $\mathrm{R} 267 \mathrm{G}$ will also play a potentially deleterious role in tumorigenesis.

BRCA1 mutations were detected in 6 primary HGSCs (40\%), 1 primary EC (100\%), and 2 primary UCs $(100 \%)$, but not in CCC $(0 \%)$ (Table 1$)$. The mutation positions in BRCA1 are shown in Fig. 2b and Supplementary Table 2 . Ten mutation positions were detected in primary tumors, and all of them were found in those reported in the public online database BRCA Exchange or ClinVar: codons 52, 63, 797, 871, 1038, 1183, 1609, 1613, 1628, and 1853 (underlined in Supplementary Table 2).

BRCA2 mutations were found in 9 primary HGSCs (60\%), 1 primary CCC (17\%), 1 primary EC (100\%), and 2 primary UCs $(100 \%)$ (Table 1$)$. The mutation positions in BRCA2 are shown in Fig. 2c and Supplementary Table 3. Fifteen mutation positions were detected in primary tumors, and eight of them were consistent with those reported in the public online database BRCA Exchange: codons 372, 819, 991, 1024, 2447, 2466, 2475, and 2729 (underlined in Supplementary Table 3). Among the new mutations detected, E911K has the highest CADD score.

ARID1A mutations were found in 3 primary CCCs $(50 \%)$ but not in primary HGSCs, EC, and UCs (Table 1). The mutation positions in ARIDIA are shown in Fig. $3 \mathrm{a}$ and Table 3. All five mutation positions detected in primary tumors were not identical to those reported in the public online databases. Among these new mutations, E2098X and R1276X with premature stop codon showed 
a

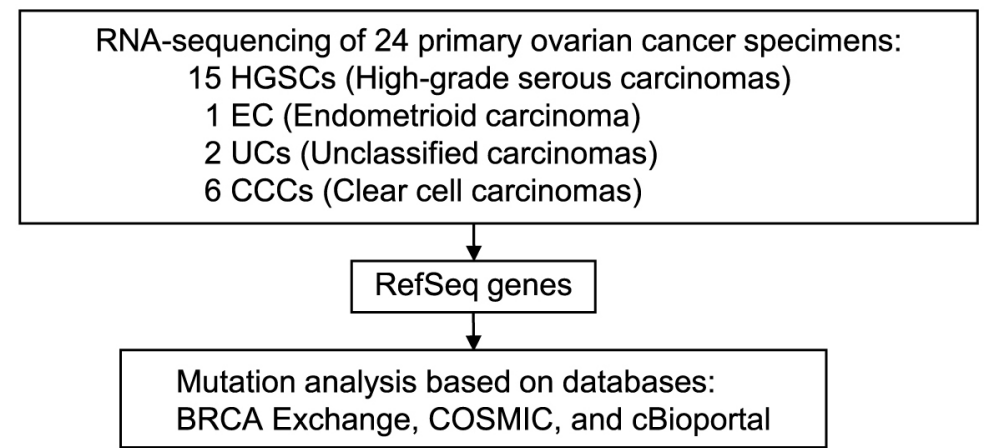

b

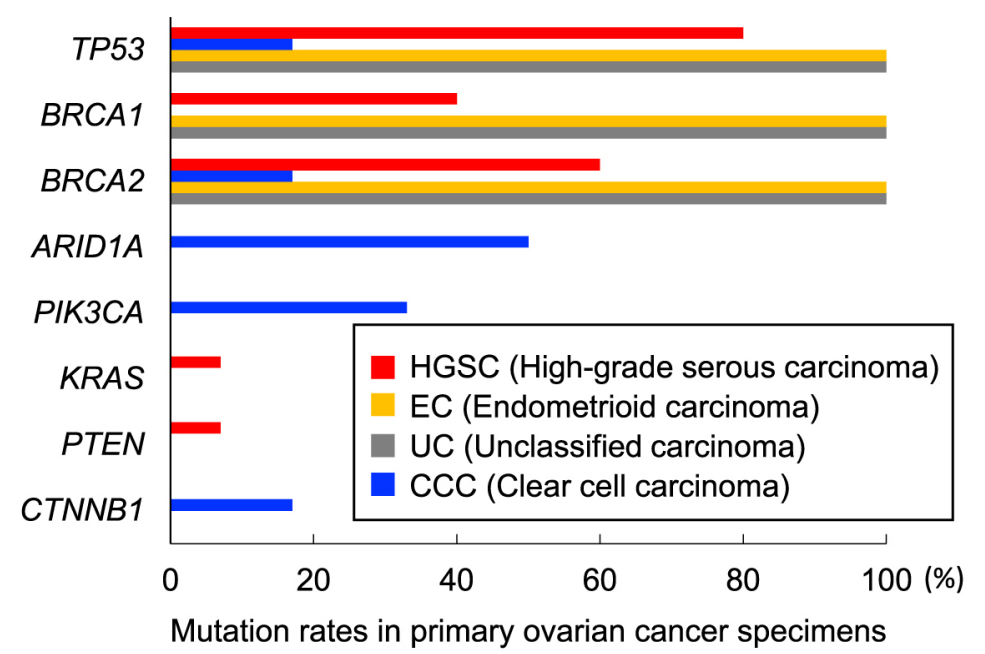

Fig. 1 Gene mutation analysis of primary ovarian cancer specimens by RNA-sequencing

(a) Schematic representation of mutation analysis in primary ovarian cancer specimens using RNA-sequencing. (b) Gene mutation rates observed in primary ovarian cancer specimens are shown in each histotype.

relatively high CADD score.

PIK3CA mutations were found in 2 primary CCCs (33\%), KRAS mutation was found in 1 primary HGSC (7\%), PTEN mutation was found in 1 primary HGSC (7\%), and CTNNB1 mutations were found in 1 primary CCC (17\%) (Table 1). These mutation positions are shown in Fig. 3b-e and upplementary Tables 4-7. One mutation position of $P I K 3 C A$ is consistent with a hotspot (H1047R) and one mutation position of CTNNB1 is registrated in ClinVar; however, mutation positions of KRAS and PTEN were not found in those reported in the public online databases.

\section{Matched-paired specimens reveal novel hotspots of Japanese patients}

Next, we performed RNA-seq using 8 recurrent ovarian cancer specimens (Sample No. S11R-S15R, E1R, $\mathrm{E} 2 \mathrm{R}$, and U1R), which are paired to the primary cancers (Sample No. S11-S15, E1, E2, and U1), and searched mutations for these genes (Supplementary Fig. 1a). The mutation rates in each histotype of recurrent tumors are shown in Supplementary Fig. 1b.
In terms of TP53 mutation, a primary mutation position $\mathrm{P} 72 \mathrm{R}$ remained in recurrent pairs of $\mathrm{E} 1, \mathrm{U} 1$, and $\mathrm{U} 2$, whereas not in those of HGSCs (Sample No. S11-S15) (Table 2). In addition, S12R, S14R, and U1R received new mutations on TP53.

BRCA1 mutations were found in 1 HGSC (Sample No. S15R), 1 EC, and 2 UCs (Sample No. U1R and U2R) (Table 1). Notably, the several primary mutation positions were retained in the recurrent tumors: P871L in E1; E1038G in E1 and U2; K1183R in E1, U1, and U2; and S1613G in E1 and U1 (Supplementary Table 2). The other primary mutation positions of BRCA1 were not detected in the recurrent specimens. Besides, S15R, $\mathrm{U} 1 \mathrm{R}$, and $\mathrm{U} 2 \mathrm{R}$ received new mutations.

$B R C A 2$ mutations were found in $3 \mathrm{HGSCs}, 1 \mathrm{EC}$, and 2 UCs (Table 1). Primary mutation position V2466A remained in the recurrent pairs of E1, U1, and U2, whereas not in those of HGSCs (S11-S15)(Supplementary Table 3 ). The other primary mutation positions were not detected in the recurrent pairs. Additionaly, S11R, S12R, S15R, U1R, and U2R received new mutation positions (Supplementary Table 3 ). 
Table 1 Number of mutations observed in each sample

\begin{tabular}{|c|c|c|c|c|c|c|c|c|c|c|}
\hline \multirow{2}{*}{ Status } & \multirow{2}{*}{ Case } & \multirow{2}{*}{ Pathology } & \multicolumn{8}{|c|}{ Gene } \\
\hline & & & TP53 & $B R C A 1$ & $B R C A 2$ & $A R I D I A$ & $P I K 3 C A$ & $K R A S$ & PTEN & $C T N N B 1$ \\
\hline \multirow[t]{24}{*}{ Primary } & $\mathrm{S} 1$ & Serous & 1 & 0 & 0 & 0 & 0 & 0 & 0 & 0 \\
\hline & $\mathrm{S} 2$ & Serous & 1 & 0 & 0 & 0 & 0 & 0 & 0 & 0 \\
\hline & $\mathrm{S} 3$ & Serous & 1 & 0 & 0 & 0 & 0 & 0 & 0 & 0 \\
\hline & S4 & Serous & 0 & 1 & 3 & 0 & 0 & 0 & 0 & 0 \\
\hline & S5 & Serous & 1 & 1 & 0 & 0 & 0 & 0 & 0 & 0 \\
\hline & S6 & Serous & 1 & 0 & 1 & 0 & 0 & 0 & 0 & 0 \\
\hline & S7 & Serous & 0 & 1 & 1 & 0 & 0 & 0 & 0 & 0 \\
\hline & S8 & Serous & 1 & 0 & 1 & 0 & 0 & 0 & 0 & 0 \\
\hline & S9 & Serous & 0 & 0 & 0 & 0 & 0 & 0 & 0 & 0 \\
\hline & $\mathrm{S} 10$ & Serous & 1 & 0 & 0 & 0 & 0 & 0 & 0 & 0 \\
\hline & S11 & Serous & 1 & 0 & 1 & 0 & 0 & 0 & 0 & 0 \\
\hline & S12 & Serous & 1 & 0 & 3 & 0 & 0 & 0 & 1 & 0 \\
\hline & S13 & Serous & 1 & 1 & 2 & 0 & 0 & 1 & 0 & 0 \\
\hline & S14 & Serous & 3 & 2 & 2 & 0 & 0 & 0 & 0 & 0 \\
\hline & S15 & Serous & 1 & 1 & 4 & 0 & 0 & 0 & 0 & 0 \\
\hline & $\mathrm{C} 1$ & Clear & 0 & 0 & 0 & 2 & 1 & 0 & 0 & 0 \\
\hline & $\mathrm{C} 2$ & Clear & 0 & 0 & 0 & 0 & 0 & 0 & 0 & 0 \\
\hline & $\mathrm{C} 3$ & Clear & 1 & 0 & 1 & 0 & 1 & 0 & 0 & 0 \\
\hline & $\mathrm{C} 4$ & Clear & 0 & 0 & 0 & 0 & 0 & 0 & 0 & 0 \\
\hline & C5 & Clear & 0 & 0 & 0 & 2 & 0 & 0 & 0 & 0 \\
\hline & C6 & Clear & 0 & 0 & 0 & 1 & 0 & 0 & 0 & 1 \\
\hline & E1 & Endometrioid & 1 & 4 & 2 & 0 & 0 & 0 & 0 & 0 \\
\hline & U1 & Unclassified & 1 & 5 & 1 & 0 & 0 & 0 & 0 & 0 \\
\hline & $\mathrm{U} 2$ & Unclassified & 1 & 2 & 1 & 0 & 0 & 0 & 0 & 0 \\
\hline \multirow[t]{8}{*}{ Recurrent } & S11R* & Serous & 0 & 0 & 1 & 0 & 0 & 0 & 0 & 0 \\
\hline & $\mathrm{S} 12 \mathrm{R} *$ & Serous & 1 & 0 & 3 & 0 & 0 & 0 & 0 & 0 \\
\hline & S13R* & Serous & 0 & 0 & 0 & 0 & 0 & 0 & 0 & 0 \\
\hline & S14R* & Serous & 1 & 0 & 0 & 0 & 0 & 0 & 0 & 0 \\
\hline & $\mathrm{S} 15 \mathrm{R}^{*}$ & Serous & 0 & 1 & 1 & 0 & 0 & 0 & 0 & 0 \\
\hline & E1R* & Endometrioid & 1 & 4 & 1 & 0 & 0 & 0 & 0 & 0 \\
\hline & U1R* & Unclassified & 2 & 3 & 2 & 2 & 0 & 0 & 0 & 0 \\
\hline & U2R* & Unclassified & 1 & 4 & 3 & 0 & 0 & 0 & 0 & 0 \\
\hline
\end{tabular}

* $\mathrm{R}$ means recurrence.

The U1R has two new mutation positions in ARID1A (Table 3). In terms of PIK3CA, KRAS, PTEN, and $C T N N B 1$, mutations were observed only in primary tumors.

\section{All matched-paired specimens of HGSCs exhibit HR deficiency}

We also searched for mutations in other 15 HR-related genes using 5 matched-paired specimens of primary and recurrent HGSCs (Supplementary Table 8). The mutation positions in HR-related genes are shown in Supplementary Table 9. ATM mutations were found in 5 primary 


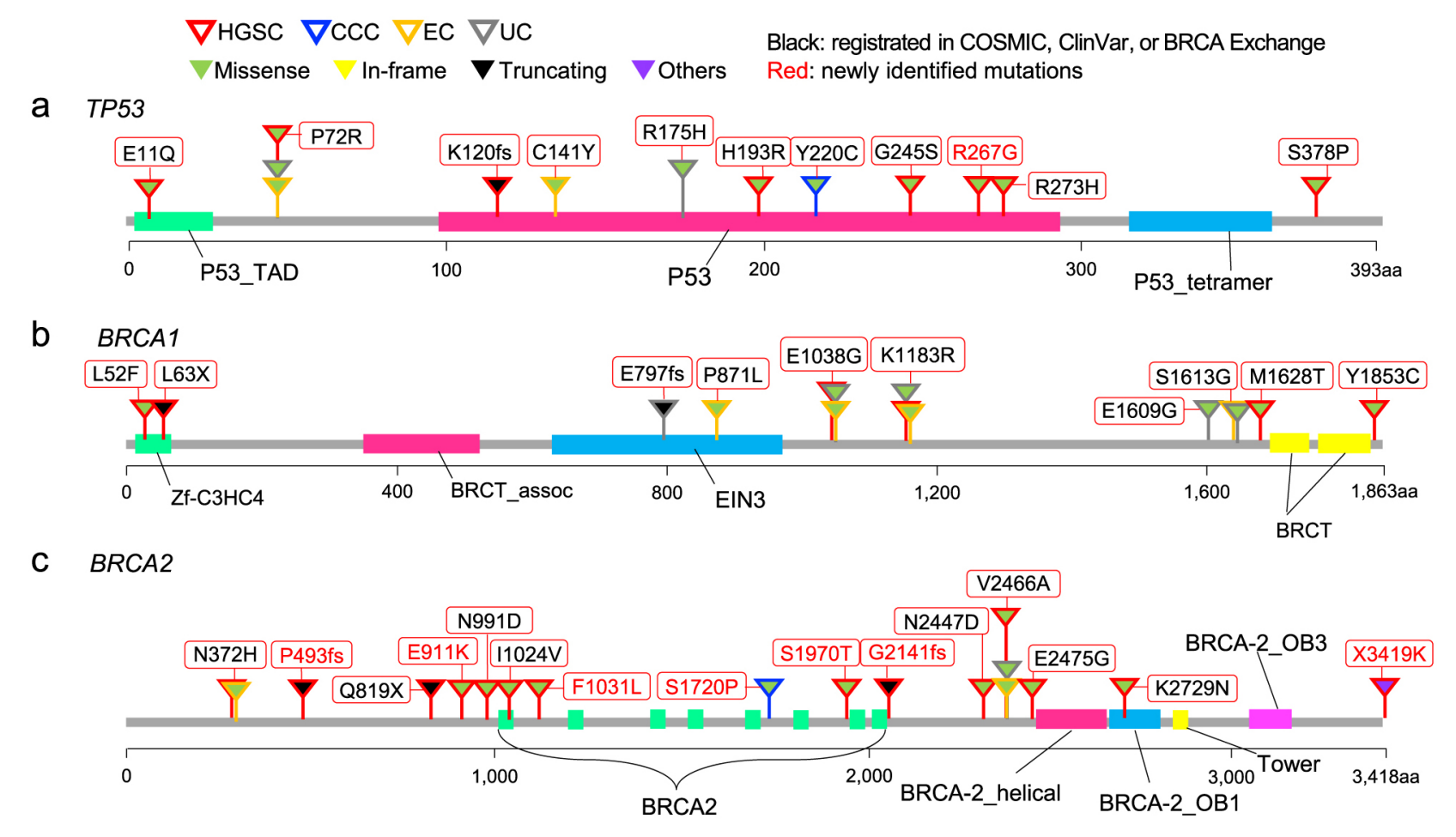

Fig. 2 Mutations detected in TP53, BRCA1, and BRCA2

Mutation positions of TP53 (a), BRCA1 (b), and BRCA2 (c) detected in primary ovarian cancer specimens are shown on the domain structures of the genes. Triangles represent mutations identified in the study where red-colored ones are newly identified mutations.

and 4 recurrent HGSCs. Among these mutations, N1983S and M1321I remained in recurrent tumors. ATR mutations were detected in 3 primary and 4 recurrent HGSCs where R2425Q and M211T mutations were found in both primary and recurrent tumors. BLM mutations were detected in 1 primary and 2 recurrent HGSCs; however, no common mutation position was found. BRIP1 mutations were found in 4 primary and 3 recurrent HGSCs. Especially, BRIP1 S919P mutation remained in recurrent pairs of S11, S13 and S14. CHEK1 I471V nonsynonymous mutations were found in all HGSCs. FANCA mutations were found in all HGSCs where G809D and G501S were retained in recurrent tumors. $N B N$ E185Q mutations were found in four pairs of primary and recurrent HGSCs. PALB2 mutations were found in 1 primary and 2 recurrent HGSCs. Three kinds of WRN mutations (A136T, L1074F, and C1367R) were found in 5 primary and 4 recurrent HGSCs. Interestingly, less than half of them was identical with those reported in the public online databases although some of them had high CADD scores (Y741X of ATM, L783P of BLM and $\mathrm{C} 42 \mathrm{X}$ of $B R I P 1)$. Among the analyzed genes, no mutation was found in CHEK2, FANCD2, FANCE, FANCF, $R A D 51 B$, and RAD51D.

\section{Discussion}

We performed mutation analysis of 24 primary ovarian cancer specimens based on RNA-sequencing and identified novel mutations in TP53, BRCA1, BRCA2, ARID1A, PIK3CA, KRAS, PTEN, and CTNNB1. Furthermore, comparison between primary and recurrent paired specimens revealed additional gene mutation in TP53, $B R C A 1, B R C A 2$, and HR-related genes. In the present study, the expression levels of above-mentioned genes were confirmed by RPKM values, which were not correlated to the detection of mutations or the numbers of mutation positions.

The tumor suppressor gene TP53 encodes the p53 protein, which plays a role in DNA repair, cell cycle arrest, apoptosis, and other pathways that prevent the development of cancers. More than $90 \%$ of HGSCs have TP53 mutation and half of them are considered to originate from the fimbriae of the fallopian tubes [23]. In the present study, $80 \%$ of primary HGSCs had TP53 mutation, whereas only $40 \%$ of recurrent HGSCs had. In comparison of paired HGSC specimens, all primary TP53 mutations were not detected in their recurrent specimens. This suggests that the HGSC specimens have heterogeneity within the same patient. In addition, cancer 
Table 2 Mutation positions of TP53

\begin{tabular}{|c|c|c|c|c|c|c|c|c|}
\hline Status & $\begin{array}{l}\text { Genome } \\
\text { position }\end{array}$ & Mutation & $\begin{array}{l}\text { Genome } \\
\text { change }\end{array}$ & $\begin{array}{c}\text { Amino acid } \\
\text { change }\end{array}$ & Exonic Function & Pathology & Subject & $\begin{array}{c}\text { CADD } \\
\text { socre }\end{array}$ \\
\hline \multirow[t]{18}{*}{ Primary } & chr17: 7579882 & $\mathrm{C}>\mathrm{G}$ & G31C & E11Q* & nonsynonymous SNV & Serous & S8 & 13.28 \\
\hline & chr17: 7579472 & $\mathrm{G}>\mathrm{C}$ & $\mathrm{C} 215 \mathrm{G}$ & $\underline{\mathrm{P} 72 \mathrm{R}^{*}}$ & nonsynonymous SNV & Serous & S11 & 8.316 \\
\hline & chr17: 7579472 & $\mathrm{G}>\mathrm{C}$ & $\mathrm{C} 215 \mathrm{G}$ & $\underline{\mathrm{P} 72 \mathrm{R}^{*}}$ & nonsynonymous SNV & Serous & $\mathrm{S} 12$ & 8.316 \\
\hline & chr17: 7579472 & $\mathrm{G}>\mathrm{C}$ & $\mathrm{C} 215 \mathrm{G}$ & $\underline{\mathrm{P}^{2} \mathrm{R}^{*}}$ & nonsynonymous SNV & Serous & $\mathrm{S} 13$ & 8.316 \\
\hline & chr17: 7579472 & $\mathrm{G}>\mathrm{C}$ & $\mathrm{C} 215 \mathrm{G}$ & $\underline{\mathrm{P} 72 \mathrm{R}^{*}}$ & nonsynonymous SNV & Serous & S14 & 8.316 \\
\hline & chr17: 7579472 & $\mathrm{G}>\mathrm{C}$ & $\mathrm{C} 215 \mathrm{G}$ & $\underline{\mathrm{P}^{2} \mathrm{R}^{*}}$ & nonsynonymous SNV & Endometrioid & E1 & 8.316 \\
\hline & chr17: 7579472 & $\mathrm{G}>\mathrm{C}$ & $\mathrm{C} 215 \mathrm{G}$ & $\underline{\mathrm{P} 72 \mathrm{R}^{*}}$ & nonsynonymous SNV & Unclassified & U1 & 8.316 \\
\hline & chr17: 7579472 & $\mathrm{G}>\mathrm{C}$ & $\mathrm{C} 215 \mathrm{G}$ & $\underline{\mathrm{P}^{2} \mathrm{R}^{*}}$ & nonsynonymous SNV & Unclassified & $\mathrm{U} 2$ & 8.316 \\
\hline & chr17: 7579328 & $\mathrm{CT}>\mathrm{C}$ & 369delA & $\underline{\mathrm{K} 120 \mathrm{fs} *}$ & frameshift deletion & Serous & S14 & —*** \\
\hline & chr17: 7578508 & $\mathrm{C}>\mathrm{T}$ & G422A & $\underline{\mathrm{C} 141 \mathrm{Y}^{*}}$ & nonsynonymous SNV & Serous & S10 & 15.06 \\
\hline & chr17: 7578406 & $\mathrm{C}>\mathrm{T}$ & G524A & $\underline{\mathrm{R} 175 \mathrm{H}^{*}}$ & nonsynonymous SNV & Serous & S3 & 31 \\
\hline & chr17: 7578406 & $\mathrm{C}>\mathrm{T}$ & G524A & $\underline{\mathrm{R} 175 \mathrm{H}^{*}}$ & nonsynonymous SNV & Serous & S5 & 31 \\
\hline & chr17: 7578271 & $\mathrm{~T}>\mathrm{C}$ & A578G & $\underline{\mathrm{H} 193 \mathrm{R}^{*}}$ & nonsynonymous SNV & Serous & S6 & 14.03 \\
\hline & chr17: 7578190 & $\mathrm{~T}>\mathrm{C}$ & A659G & $\underline{\mathrm{Y} 220 \mathrm{C}^{*}}$ & nonsynonymous SNV & Clear & $\mathrm{C} 3$ & 22.1 \\
\hline & chr17: 7577548 & $\mathrm{C}>\mathrm{T}$ & G733A & $\underline{\mathrm{G} 245 \mathrm{~S}^{*}}$ & nonsynonymous SNV & Serous & S2 & 33 \\
\hline & chr17: 7577139 & $\mathrm{G}>\mathrm{C}$ & C799G & $\mathrm{R} 267 \mathrm{G}$ & nonsynonymous SNV & Serous & S15 & 20.5 \\
\hline & chr17: 7577120 & $\mathrm{C}>\mathrm{T}$ & G818A & $\underline{\mathrm{R} 273 \mathrm{H}^{*}}$ & nonsynonymous SNV & Serous & S1 & 18.03 \\
\hline & chr17: 7572977 & $A>G$ & $\mathrm{~T} 1132 \mathrm{C}$ & $\underline{\mathrm{S} 378 \mathrm{P} *}$ & nonsynonymous SNV & Serous & S14 & 14.01 \\
\hline \multirow[t]{6}{*}{ Recurrent } & chr17: 7579472 & $\mathrm{G}>\mathrm{C}$ & $\mathrm{C} 215 \mathrm{G}$ & $\underline{\mathrm{P} 72 \mathrm{R}^{*}}$ & nonsynonymous SNV & Endometrioid & $\mathrm{E} 1 \mathrm{R}^{* *}$ & 8.316 \\
\hline & chr17: 7579472 & $\mathrm{G}>\mathrm{C}$ & $\mathrm{C} 215 \mathrm{G}$ & $\underline{\mathrm{P} 72 \mathrm{R}^{*}}$ & nonsynonymous SNV & Unclassified & $\mathrm{U} 1 \mathrm{R}^{* *}$ & 8.316 \\
\hline & chr17: 7579472 & $\mathrm{G}>\mathrm{C}$ & $\mathrm{C} 215 \mathrm{G}$ & $\underline{\mathrm{P} 72 \mathrm{R}^{*}}$ & nonsynonymous SNV & Unclassified & $\mathrm{U} 2 \mathrm{R}^{* *}$ & 8.316 \\
\hline & chr17: 7578490 & $\mathrm{AC}>\mathrm{A}$ & 439delG & V147fs & frameshift deletion & Unclassified & $\mathrm{U} 1 \mathrm{R}^{* *}$ & —*** \\
\hline & chr17: 7577521 & $\mathrm{TG}>\mathrm{T}$ & 759delC & T253fs & frameshift deletion & Serous & $\mathrm{S} 12 \mathrm{R} * *$ & —*** \\
\hline & chr17: 7574003 & $\mathrm{G}>\mathrm{A}$ & C1024T & $\underline{\mathrm{R} 342 \mathrm{X}^{*}}$ & stopgain & Serous & S14R** & 37 \\
\hline
\end{tabular}

* Underline means registrated in COSMIC or ClinVar.

** $\mathrm{R}$ means recurrence.

*** - means there is no CADD score.

cells containing such mutations may be eradicated by therapy. In contrast, UC and EC retained their primary TP53 mutation (P72R) in the recurrent specimens, suggesting a hotspot mutation of Japanese UC and EC patients.

Generally, BRCA germline mutations are observed in $13 \%-16 \%$ and somatic mutations are detected in approximately $6 \%$ of HGSCs [24]. The hallmarks of BRCA1/ $B R C A 2$-associated ovarian carcinomas include sensitivity to platinum chemotherapy, improvement of overall survival [25-30], and sensitivity to poly(ADP-ribose) polymerase inhibitors (PARP-i) $[31,32]$. In addition to BRCA1/BRCA2 mutations, other HR-related gene mutations may increase sensitivity to DNA-damaging agents. In vitro studies demonstrate that deficiency in other HR proteins such as ATM, CHEK1, CHEK2, NBN, and
RAD51D also confer sensitivity to PARP-i [33, 34]. PARP-i are active agents in ovarian carcinomas with germline $B R C A 1 / B R C A 2$ mutations but also in a subset of "sporadic" recurrent platinum-sensitive ovarian carcinomas [32]. Indeed, TCGA reported HR defects in approximately $50 \%$ of HGSCs [6]. In the present study, $40 \%$ of primary HGSCs and $20 \%$ of recurrent HGSCs possessed $B R C A 1$ mutation, and $60 \%$ of primary HGSCs and $60 \%$ of recurrent HGSCs showed BRCA2 mutation. Notably, the incident of $B R C A$ mutation was much higher among Japanese women than those reported from Western countries. Sugano et al. reported the possibility of higher incident of $B R C A$ mutation among Japanese patients compared to non-Ashkenazi individuals in the US [35]. All of the primary BRCA1 and BRCA2 mutations of HGSCs were not detected in the recurrent speci- 


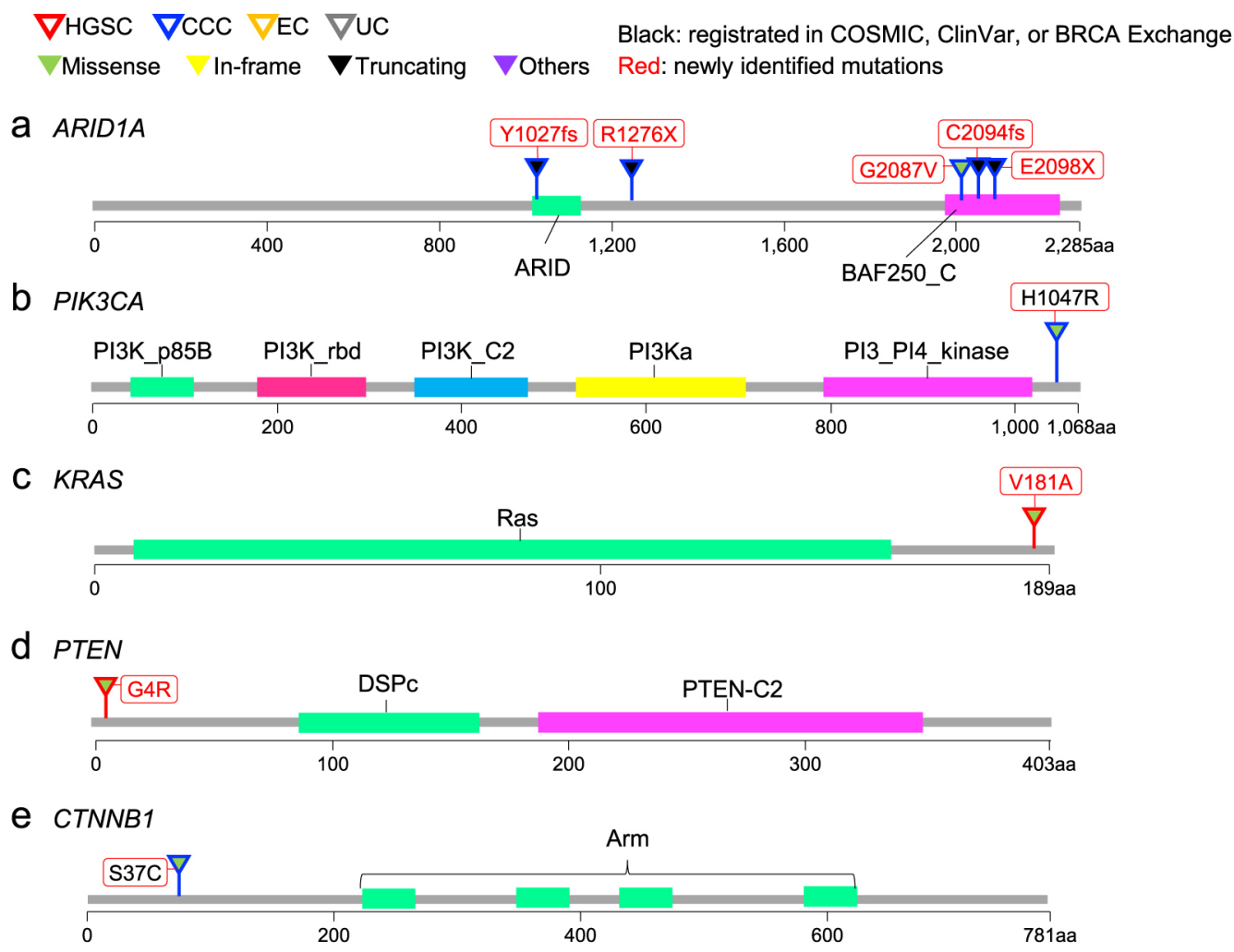

Fig. 3 Mutations detected in ARIDIA, PIK3CA, KRAS, PTEN, and CTNNB1

Mutation positions of ARIDIA (a), PIK3CA (b), KRAS (c), PTEN (d), and CTNNB1 (e) detected in primary ovarian cancer specimens are shown on the domain structures of the genes. Triangles represent mutations identified in the study where redcolored ones are newly identified mutations.

Table 3 Mutation positions of ARIDIA

\begin{tabular}{|c|c|c|c|c|c|c|c|c|}
\hline Status & $\begin{array}{l}\text { Genome } \\
\text { position }\end{array}$ & Mutation & $\begin{array}{l}\text { Genome } \\
\text { change }\end{array}$ & $\begin{array}{c}\text { Amino acid } \\
\text { change }\end{array}$ & ExonicFunction & Pathology & Subject & $\begin{array}{l}\text { CADD } \\
\text { score }\end{array}$ \\
\hline \multirow[t]{5}{*}{ Primary } & chr1: 27094372 & $\mathrm{AT}>\mathrm{A}$ & 3081 delT & Y1027fs & frameshift deletion & Clear & $\mathrm{C} 1$ & —** \\
\hline & chr1: 27099947 & $\mathrm{C}>\mathrm{T}$ & $\mathrm{C} 3826 \mathrm{~T}$ & R1276X & stop gain & Clear & $\mathrm{C} 5$ & 41 \\
\hline & chr1: 27106649 & $\mathrm{G}>\mathrm{T}$ & G6260T & G2087V & nonsynonymous SNV & Clear & C6 & 17.09 \\
\hline & chr1: 27106670 & $\mathrm{GC}>\mathrm{G}$ & $6282 \mathrm{delC}$ & C2094fs & frameshift deletion & Clear & $\mathrm{C} 5$ & —** \\
\hline & chr1: 27106681 & $\mathrm{G}>\mathrm{T}$ & G6292T & E2098X & stop gain & Clear & $\mathrm{C} 1$ & 43 \\
\hline \multirow[t]{2}{*}{ Recurrent } & chr1: 27023063 & $\mathrm{GA}>\mathrm{G}$ & 170delA & E57fs & frameshift deletion & Unclassified & U1R* & —** \\
\hline & chr1: 27023085 & $\mathrm{G}>\mathrm{A}$ & G191A & G64E & nonsynonymous SNV & Unclassified & U1R* & 17.07 \\
\hline
\end{tabular}

* $\mathrm{R}$ means recurrence.

** _ means there is no CADD score.

mens. In contrast, all UCs and ECs retained one primary BRCA1 mutation (K1183R) and one primary BRCA2 mutation (V2466A). These results suggest that primary HGSC with BRCA1 or BRCA2 mutation is sufficiently controlled by the standard first-line therapy and that tumors without $B R C A$ mutation exhibit recurrence. However, primary HGSC specimens retained their HR-related gene mutations after recurrence: ATM, ATR, BRIP1, CHEK1, FANCA, NBN, PALB2, and WRN. Therefore, in recurrent HGSCs not retaining $B R C A 1$ or $B R C A 2$ mutations, treatment using PARP-i may be effective due to their HR-defect status. Additionally, mutations of L63X in $B R C A 1$ among Japanese ovarian cancer patients, and mutations of M1628T in BRCA1 and $\mathrm{K} 2729 \mathrm{~N}$ in BRCA2 among Korean ovarian cancer patients were previously reported $[35,36]$. The findings of this study suggest that L63X, M1628T, K1183R, and L771L may be new hotspots of BRCA1 mutations, and L1521L, V2466A, and 
K2729N may be new hotspots of BRCA2 mutations in East Asian patients.

Regarding CCCs, the main driver mutations are assumed to be the loss-of-function of ARIDIA or the activation of the phosphoinositol 3-kinase (PI3K) pathway [37-40]. We observed the proportion of ARID1A in $50 \%$ CCCs and PIK3CA mutations in 33\% CCCs. Two patients exhibited ARID1A mutation, one patient exhibited $P I K 3 C A$ mutation, and the other one patient exhibited both of these mutations. Wiegand et al. found that $46 \%$ of patients with CCC had somatic truncating or missense mutations of ARIDIA [37]. ARIDIA encodes the protein BAF250a, which acts as an accessory subunit of the SWItch/Sucrose Non-Fermentable chromatin remodeling complex, which regulates several cellular processes, including tumor suppression. ARIDIA mutations result in the functional absence or decreased expression of BAF250a. Endometriotic lesions that are adjacent to endometrioid lesions also exhibit a loss-offunction BAF250a mutation, suggesting that this mutation may be an early step in the transformation [38]. PIK3CA encodes for $\mathrm{p} 110 \mathrm{a}$, which forms the catalytic subunit of the phosphorylation molecule PI3K [39]. PIK3CA mutations were found to be present in $40 \%$ of patients with CCCs and comprised missense and cluster mutations in exons 9 and 20. The activation of the PI3K/AKT pathway regulates downstream target genes that inhibit apoptosis and promote cell proliferation and also opposes the action of PTEN, a tumor suppressor that acts as a negative regulator for the aforementioned pathway [40].

Atypical endometriosis is a direct precursor of EC, with $40 \%$ of carcinomas presenting concurrent endometriosis [41]. EC is likely to be found at an early stage and has been reported to have somatic mutations of CTNNB1, PTEN, and PIK3CA [42]. CTNNB1 is a gene that encodes $\beta$-catenin, a protein that plays an important role in cell adhesion and cell proliferation [43]. CTNNB1 mutations lead to squamous cell differentiation, which is a histopathologic feature of endometrioid cancers [42]. However, in this study, CTNNB1 mutations were detected only in one primary CCC but not in EC. KRAS and $P T E N$ mutations were detected in one primary HGSC but not in EC. In the present study, the mutation rates and their common histotypes of KRAS, PTEN, and CTNNB1 were different from the existing data mainly based on Western countries.

The majority of mutations observed in HR-related genes were not detected in the public online database COSMIC. Notably, in this study, we newly identified 3 mutation positions in ATM, 3 in ATR, 2 in BLM, 2 in BRIP1, 3 in FANCA, 1 in PALB2, and 2 in $W R N$. We identified the possible new hotspots of $A T M$ (N1983S),
ATR (M211T and R2425Q), BRIP1 (S919P), CHEK1 (I471V), FANCA (T266A, G501S, and G809D), NBN (E185Q), and WRN (L1074F).

Unlike DNA sequencing, RNA-sequencing provides the information for transcripts after RNA processing and enables to analyze simultaneously both gene mutation and expression. The data generated by RNA-sequencing have advantages to more easily identify allele-specific expression, alternatively spliced genes, and non-coding RNAs. Therefore, RNA-sequencing is capable of uncovering novel mutations in transcriptionally active genes. Sun et al. reported that RNA-sequencing based on commonly used analysis programs with variant and somatic mutation callers can be reliably used for both single nucleotide variation (SNV) and indel mutation detection that can be used for clinical management [44]. Nevertheless, there are limitations of RNA-sequencing for analyzing transcriptionally unexpressed genes and posttranscriptionally silenced genes exemplified by nonsense-mediated mRNA decay. Based on a comparison study of exome-sequencing and RNA-sequencing for lung cancer samples by O'Brien et al., they discussed the differences of the two methods and discrepancies in SNV calling [45]. While SNVs detected by exome-sequencing are derived from captured exon regions, RNA-sequencing could also detect SNVs outside the boundary of the exome-seq capture regions. SNVs detected by RNAsequencing also show mutational signature of RNA editing especially in 3'-untranslated region, as exemplified by the deamination of adenosine that coverts to inosine, resulting in adenosine to guanine conversion. In the present study, we consider that RNA editing would have minimal contribution to the development of novel variants, as we basically focus on coding regions of cancerrelated genes. The results by O'Brien et al. indicate that RNA-sequencing could be also a practical technology platform to detect SNVs together with transcript expression, while we need to remind that some SNVs detected by RNA-sequencing would be unique and not observed in exome-seq [45].

The present mutation study based on RNA-sequencing showed that 13 of 14 mutations in TP53, 10 of 11 mutations of $B R C A 1,10$ of 23 mutation positions of $B R C A 2$, none of 7 mutations of ARIDIA, 1 mutation of PIK3CA, and 1 mutation of $C T N N B 1$ were consistent with those reported in the public online databases, which were basically constructed by exome-sequencing. This suggests the reliability of mutation analysis using RNAsequencing data. Notably, a number of novel mutations were identified as putative specific gene alterations in Japanese patients. We examined CADD score for each mutation and found that some of the novel mutations we identified have high CADD score, suggesting their 
pathological roles in ovarian cancer. Nevertheless, the precise clinical relevance for each mutation remains to be clarified, thus we may not exclude potential benign variants and variants of unknown significance from the gene mutation list in the present study. Moreover, future gain/loss-of-function study of genes with differential expression will help in further elucidating histotypespecific molecular mechanisms. RNA-sequencing-based mutation analysis is useful to determine the contribution of gene mutation in ovarian cancer and to understand ethnic differences in mutations.

\section{Acknowledgements}

We thank Mr. W Sato, Mr. T Oishi, and Ms. A Iwasa for their technical assistance. This work was partially supported by Grant of the Support Project of Strategic Research Center in Private Universities from the Ministry of Education, Culture, Sports, Science, and Technology, Japan; by the Practical Research for Innovative Cancer Control (JP18ck0106194) and the Project for Cancer Research And Therapeutic Evolution (PCREATE, JP18cm0106144) from Japan Agency for Medical Research and development, AMED; by Grants from the Japan Society for the Promotion of Science, Japan (16K09809, 16K15496, 17H04205); by the Takeda Science Foundation.

\section{Disclosure}

The authors declare that they have no competing interests.

\section{References}

1. Center for Cancer Control and Information Services, National Cancer Canter, Japan. Projected Cancer Statistics, 2018. (https://ganjoho.jp/en/public/statistics/short_pred.html)

2. Gurung A, Hung T, Morin J, Gilks CB (2013) Molecular abnormalities in ovarian carcinoma: clinical, morphological and therapeutic correlates. Histopathology 62: 59-70.

3. Reid BM, Permuth JB, Sellers TA (2017) Epidemiology of ovarian cancer: a review. Cancer Biol Med 14: 9-32.

4. Yamagami W, Nagase S, Takahashi F, Ino K, Hachisuga $\mathrm{T}$, et al. (2017) Clinical statistics of gynecologic cancers in Japan. J Gynecol Oncol 28: e32.

5. Weinstein IB (2002) Cancer. Addiction to oncogenes-the Achilles heal of cancer. Science 297: 63-64.

6. Cancer Genome Atlas Research Network (2011) Integrated genomic analyses of ovarian carcinoma. Nature 474: 609-615.

7. Hayano T, Yokota Y, Hosomichi K, Nakaoka H, Yoshihara K, et al. (2014) Molecular characterization of an intact p53 pathway subtype in high-grade serous ovarian cancer. PLoS One 9: e114491.

8. Hirasawa A, Imoto I, Naruto T, Akahane T, Yamagami W, et al. (2017) Prevalence of pathogenic germline variants detected by multigene sequencing in unselected Japanese patients with ovarian cancer. Oncotarget 8: 112258 112267.

9. Enomoto T, Aoki D, Hattori K, Jinushi M, Kigawa J, et al. (2019) The first Japanese nationwide multicenter study of BRCA mutation testing in ovarian cancer: CHARacterizing the cross-sectionaL approach to Ovarian cancer geneTic TEsting of BRCA (CHARLOTTE). Int J Gynecol Cancer 29: 1043-1049.

10. Pearce CL, Templeman C, Rossing MA, Lee A, Near AM, et al. (2012) Association between endometriosis and risk of histological subtypes of ovarian cancer: a pooled analysis of case-control studies. Lancet Oncol 13: 385-394.
11. Mabuchi S, Sugiyama T, Kimura T (2016) Clear cell carcinoma of the ovary: molecular insights and future therapeutic perspectives. J Gynecol Oncol 27: e31.

12. Shibuya Y, Tokunaga H, Saito S, Shimokawa K, Katsuoka $\mathrm{F}$, et al. (2018) Identification of somatic genetic alterations in ovarian clear cell carcinoma with next generation sequencing. Genes Chromosomes Cancer 57: 51-60.

13. Ishikawa M, Nakayama K, Nakamura K, Ono R, Sanuki $\mathrm{K}$, et al. (2018) Affinity-purified DNA-based mutation profiles of endometriosis-related ovarian neoplasms in Japanese patients. Oncotarget 9: 14754-14763.

14. Cho KR, Shih IeM (2009) Ovarian cancer. Annu Rev Pathol 4: 287-313.

15. Gadducci A, Lanfredini N, Tana R (2014) Novel insights on the malignant transformation of endometriosis into ovarian carcinoma. Gynecol Endocrinol 30: 612-617.

16. Gemignani ML, Schlaerth AC, Bogomolniy F, Barakat $\mathrm{RR}$, Lin O, et al. (2003) Role of KRAS and BRAF gene mutations in mucinous ovarian carcinoma. Gynecol Oncol 90: 378-381.

17. Mackenzie R, Kommoss S, Winterhoff BJ, Kipp BR, Garcia JJ, et al. (2015) Targeted deep sequencing of mucinous ovarian tumors reveals multiple overlapping RASpathway activating mutations in borderline and cancerous neoplasms. BMC Cancer 15: 415.

18. Takenaka M, Saito M, Iwakawa R, Yanaihara N, Saito M, et al. (2015) Profiling of actionable gene alterations in ovarian cancer by targeted deep sequencing. Int $J$ Oncol 46: 2389-2398.

19. Patel JN, Braicu I, Timms KM, Solimeno C, Tshiaba P, et al. (2018) Characterisation of homologous recombination deficiency in paired primary and recurrent high-grade serous ovarian cancer. Br J Cancer 119: 1060-1066.

20. Li C, Bonazzoli E, Bellone S, Choi J, Dong W, et al. (2019) Mutational landscape of primary, metastatic, and 
recurrent ovarian cancer reveals c-MYC gains as potential target for BET inhibitors. Proc Natl Acad Sci U S A 116: 619-624.

21. Sakai W, Swisher EM, Karlan BY, Agarwal MK, Higgins $\mathrm{J}$, et al. (2008) Secondary mutations as a mechanism of cisplatin resistance in BRCA2-mutated cancers. Nature 451: 1116-1120.

22. Kircher M, Witten DM, Jain P, O'Roak BJ, Cooper GM, et al. (2014) A general framework for estimating the relative pathogenicity of human genetic variants. Nat Genet 46: 310-315.

23. Gurung A, Hung T, Morin J, Gilks CB (2013) Molecular abnormalities in ovarian carcinoma: clinical, morphological and therapeutic correlates. Histopathology 62: 59-70.

24. Shaw PA, McLaughlin JR, Zweemer RP, Narod SA, Risch $\mathrm{H}$, et al. (2002) Histopathologic features of genetically determined ovarian cancer. Int J Gynecol Pathol 21: 407411.

25. Alsop K, Fereday S, Meldrum C, deFazio A, Emmanuel C, et al. (2012) BRCA mutation frequency and patterns of treatment response in BRCA mutation-positive women with ovarian cancer: a report from the Australian Ovarian Cancer Study Group. J Clin Oncol 30: 2654-2663.

26. Bolton KL, Chenevix-Trench G, Goh C, Sadetzki S, Ramus SJ, et al. (2012) Association between BRCA1 and BRCA2 mutations and survival in women with invasive epithelial ovarian cancer. JAMA 307: 382-390.

27. Chetrit A, Hirsh-Yechezkel G, Ben-David Y, Lubin F, Friedman E, et al. (2008) Effect of BRCA1/2 mutations on long-term survival of patients with invasive ovarian cancer: the national Israeli study of ovarian cancer. J Clin Oncol 26: 20-25.

28. Tan DS, Rothermundt C, Thomas K, Bancroft E, Eeles R, et al. (2008) "BRCAness" syndrome in ovarian cancer: a case-control study describing the clinical features and outcome of patients with epithelial ovarian cancer associated with BRCA1 and BRCA2 mutations. J Clin Oncol 26: 5530-5536.

29. Vencken PM, Kriege M, Hoogwerf D, Beugelink S, van der Burg ME, et al. (2011) Chemosensitivity and outcome of BRCA1- and BRCA2-associated ovarian cancer patients after first-line chemotherapy compared with sporadic ovarian cancer patients. Ann Oncol 22: 13461352.

30. Yang D, Khan S, Sun Y, Hess K, Shmulevich I, et al. (2011) Association of BRCA1 and BRCA2 mutations with survival, chemotherapy sensitivity, and gene mutator phenotype in patients with ovarian cancer. JAMA 306: $1557-$ 1565.

31. Audeh MW, Carmichael J, Penson RT, Friedlander M, Powell B, et al. (2010) Oral poly(ADP-ribose) polymerase inhibitor olaparib in patients with BRCA1 or BRCA2 mutations and recurrent ovarian cancer: a proof-ofconcept trial. Lancet 376: 245-251.

32. Gelmon KA, Tischkowitz M, Mackay H, Swenerton K,
Robidoux A, et al. (2011) Olaparib in patients with recurrent high-grade serous or poorly differentiated ovarian carcinoma or triplenegative breast cancer: a phase 2, multicentre, open-label, non-randomised study. Lancet Oncol 12: 852-861.

33. Loveday C, Turnbull C, Ramsay E, Hughes D, Ruark E, et al. (2011) Germline mutations in RAD51D confer susceptibility to ovarian cancer. Nat Genet 43: 879-882.

34. McCabe N, Turner NC, Lord CJ, Kluzek K, Bialkowska A, et al. (2006) Deficiency in the repair of DNA damage by homologous recombination and sensitivity to poly (ADP-ribose) polymerase inhibition. Cancer Res 66: 8109-8115.

35. Sugano K, Nakamura S, Ando J, Takayama S, Kamata H, et al. (2008) Cross-sectional analysis of germline BRCA1 and BRCA2 mutations in Japanese patients suspected to have hereditary breast/ovarian cancer. Cancer Sci 99: 1967-1976.

36. Choi MC, Jang JH, Jung SG, Park H, Joo WD, et al. (2018) Unclassified variants of BRCA1 and BRCA2 in Korean patients with ovarian cancer. Int J Gynecol Cancer 28: $308-315$.

37. Wiegand KC, Shah SP, Al-Agha OM, Zhao Y, Tse K, et al. (2010) ARID1A mutations in endometriosis associated ovarian carcinomas. $N$ Engl J Med 363: 1532-1543.

38. Worley MJ Jr, Liu S, Hua Y, Kwok JS, Samuel A, et al. (2015) Molecular changes in endometriosis-associated ovarian clear cell carcinoma. Eur J Cancer 51: 18311842.

39. Kuo KT, Mao TL, Jones S, Veras E, Ayhan A, et al. (2009) Frequent activating mutations of PIK3CA in ovarian clear cell carcinoma. Am J Pathol 174: 1597-1601.

40. Rahman M, Nakayama K, Rahman MT, Nakayama N, Ishikawa M, et al. (2012) Clinicopathologic and biological analysis of PIK3CA mutation in ovarian clear cell carcinoma. Hum Pathol 43: 2197-2206.

41. Terada T (2012) Endometrioid adenocarcinoma of the ovary arising in atypical endometriosis. Int J Clin Exp Pathol 5: 924-927.

42. Kurman RJ, Shih IeM (2011) Molecular pathogenesis and extraovarian origin of epithelial ovarian cancer-Shifting the paradigm. Hum Pathol 42: 918-931.

43. Guan L, Zhu S, Han Y, Yang C, Liu Y, et al. (2018) Knockout of CTNNB1 by CRISPR-Cas9 technology inhibits cell proliferation through the Wnt/ $\beta$-catenin signaling pathway. Biotechnol Lett 40: 501-508.

44. Sun Z, Bhagwate A, Prodduturi N, Yang P, Kocher JA (2017) Indel detection from RNA-seq data: tool evaluation and strategies for accurate detection of actionable mutations. Brief Bioinform 18: 973-983.

45. O'Brien TD, Jia P, Xia J, Saxena U, Jin H, et al. (2015) Inconsistency and features of single nucleotide variants detected in whole exome sequencing versus transcriptome sequencing: a case study in lung cancer. Methods 83: 118127. 